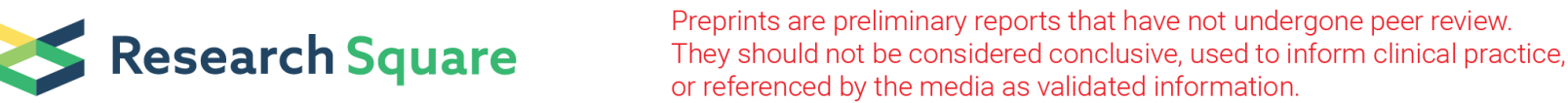

\section{Verification of Effect of Acquisition Time for SwiftScan on Quantitative Bone Single-Photon Emission Computed Tomography Using an Anthropomorphic Phantom}

Takuro Shiiba ( $\nabla$ takuro.shiiba@fujita-hu.ac.jp )

Fujita Health University https://orcid.org/0000-0001-5515-091X

\section{Yuya Sekikawa}

Teikyo University: Teikyo Daigaku

Shinji Tateoka

Koga General Hospital

Nobutaka Shinohara

Koga General Hospital

Yuuki Inoue

Koga General Hospital

Yasuyoshi Kuroiwa

Koga General Hospital

Takashi Tanaka

Koga General Hospital

\section{Yasushi Kihara}

Koga General Hospital

Takuroh Imamura

Koga General Hospital

\section{Research Article}

Keywords: SwiftScan SPECT, Bone, Quantitative SPECT, Acquisition time, Continuous step and shoot

Posted Date: January 17th, 2022

DOI: https://doi.org/10.21203/rs.3.rs-1186401/v1

License: (c) (1) This work is licensed under a Creative Commons Attribution 4.0 International License. Read Full License 


\section{Abstract}

Background: SwiftScan (GE Healthcare) is a recently developed scanning technique with data acquisition during detector static and rotation. The influence of image quality using SwiftScan on quantitative bone single-photon emission computed tomography (SPECT) remains unclear. This study clarifies the effect of the acquisition time for SwiftScan on the image quality and quantification of bone SPECT compared to step and shoot mode (SSM) using ${ }^{99 \mathrm{~m}}$ Tc-filled anthropomorphic phantom (SIM ${ }^{2}$ bone phantom).

Results: The coefficient of variance (CV), contrast-to-noise ratio (CNR), full width at half maximum (FWHM), and recovery coefficient (RC) of SwiftScan and SSM were recorded at various overall acquisition times $(5,7,17$, and $32 \mathrm{~min}$ ) when the fixed reconstruction parameters (subsets 10 and iterations 5) were compared. The CV of SSM was higher than that of SwiftScan in short-time acquisition (less than $7 \mathrm{~min}$ ), whereas the CV of SSM and SwiftScan are equivocal in long-time acquisition (more than $17 \mathrm{~min}$ ). The CNR of SwiftScan was higher than that of SSM in short-time acquisition, whereas the CNR of SSM was equivocal or higher than that of SwiftScan in long-time acquisition. The FWHM of SSM (15.2-16.5 mm) and SwiftScan (15.4-15.9 mm) was almost constant when the acquisition time was 7 min or longer. However, SwiftScan $(22.6 \mathrm{~mm})$ and SSM $(18.6 \mathrm{~mm})$ showed high FWHM values at 5 min acquisition, deviating from the actual size $(10 \mathrm{~mm})$. For the RC of SwiftScan and SSM, was no evident difference between them was observed.

Conclusions: SwiftScan on quantitative bone SPECT provides improved CV and CNR in short-time acquisition (less than $7 \mathrm{~min}$ ) with quantitativeness similar to SSM. Therefore, using SwiftScan in clinical settings instead of the SSM scan protocol in short-time acquisition (less than $7 \mathrm{~min}$ ) might provide higher quality diagnostic images than SSM. Our results would provide important information on the use of SwiftScan.

\section{Background}

Bone scintigraphy is used to detect bone metastases, such as in prostate cancer [1] and breast cancer [2]. In prostate cancer, bone scintigraphy has a high sensitivity of bone metastasis, thereby making it one of the most valuable methods to detect bone metastasis [1]. Knowing whether bone metastasis is present is essential in determining the cancer stage, effective treatment, and prognosis of such diseases [3].

In bone scintigraphy, single-photon emission computed tomography (SPECT) is used with whole-body imaging to facilitate three-dimensional positioning and improve the detection rate of lesions. Recently, combined SPECT systems with computed tomography (CT) have become widely available, and improved the accuracy of the bone lesion diagnosis by SPECT/CT imaging has been reported [4-7].

Quantification of ${ }^{99 \mathrm{~m}} \mathrm{Tc}$ bone SPECT/CT is becoming feasible as a diagnostic tool and as a means of monitoring treatment efficacy [8]. Phantom and clinical studies have shown that the quantitative accuracy of ${ }^{99 \mathrm{~m}}$ Tc-based SPECT imaging is within $\pm 10 \%$ [9]. However, for the quantification of bone SPECT imaging to become a standard clinical diagnostic method, the accumulation of more reliable 
quantitative data is needed. The accuracy of bone SPECT quantification is affected by the acquisition method, resolution, reconstruction method, and cross-calibration method of the device [10-13]. Acquisition methods, such as the step and shoot mode (SSM) and continuous mode (CM) [14], are commonly used for SPECT. SwiftScan (GE Healthcare, Milwaukee, WI, USA) [15-18] SPECT is a recently developed scanning technique with data acquisition during detector static and rotation. Thibault et al. [17] reported that the GE-developed SwiftScan with low-energy high-resolution and sensitivity (LEHRS) collimator improved sensitivity compared to that of low-energy high-resolution (LEHR) collimator. Shibutani et al.[18] reported that the SwiftScan planar and SPECT with LEHRS collimator have high sensitivity while maintaining the spatial resolution compared with the conventional system. Several researchers have shown the potential for reduced acquisition time with SwiftScan SPECT in a clinical study. Bailly et al. [16] and Picone et al. [19] showed the potential for a $25 \%$ reduction in acquisition time without degradation of image quality and quantitation in bone and lung and dopamine transporter, respectively. However, there was no investigation of the effect of acquisition time in SwiftScan using a phantom in quantitative bone SPECT. This study clarifies the effect of acquisition time for SwiftScan on the image quality and quantitative value of quantitative bone SPECT compared to SSM.

\section{Methods}

\section{Phantom}

The SIM² Bone Phantom (Kyoto Kagaku, Kyoto, Japan) [18, 20] was used. This phantom can reproduce tumor bone area in the vertebral body with four different diameters $(13,17,22$, and $28 \mathrm{~mm})$, and it has the whole vertebral body as a reference part (diameter and length of 36 and $35 \mathrm{~mm}$, respectively) (Fig. 1). The

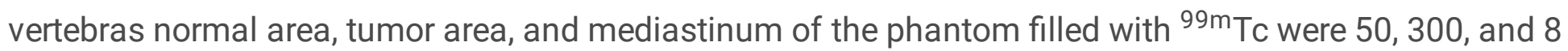
$\mathrm{kBq} / \mathrm{mL}$, respectively. The lung insert was not filled with radioactive material.

\section{Acquisition and reconstruction of SPECT/CT imaging}

The phantom SPECT/CT image acquisitions were performed using Discovery NM/CT 860 (GE Healthcare) with a LEHRS collimator. For the dual-energy window scatter correction (SC) method (scatter weighting factor was 1.10), the primary and sub-energy window acquisitions were $140 \mathrm{keV} \pm 10 \%$ and $120 \mathrm{keV} \pm 5 \%$, respectively. The matrix size for the acquisition was $128 \times 128$, the magnification ratio was 1.0 , the pixel size was $4.42 \mathrm{~mm}$, the projection number was 60 , automatic proximity was used, and the images were acquired with SwiftScan and SSM. The acquisition time was 6, 10, 30, 60 s/view for SSM. The acquisition time for SwiftScan is added to the acquisition time for the detector to move (approximately $4 \mathrm{~s}$ ) to the acquisition time of SSM. The acquisition time per projection of SwiftScan is 4 s longer than SSM; however, the overall acquisition time is equivalent. The overall acquisition times for SwiftScan and SSM were $5 \min 4 \mathrm{~s}, 7 \min 4 \mathrm{~s}, 17 \min 4 \mathrm{~s}$, and $32 \min 4 \mathrm{~s}$. CT for attenuation correction (AC) was acquired at $120 \mathrm{kV}$ and $30 \mathrm{~mA}$ with a $512 \times 512$ matrix, 1.675 pitch, and 1.0 s rotation. CT 
images were reconstructed at a $1.25-\mathrm{mm}$ slice thickness with adaptive statistical iterative reconstruction (SS80 Slice 80\%).

All acquired projection images were reconstructed using a three-dimensional iterative ordered subset expectation maximization algorithm considering the CT-based AC, dual-energy SC, and resolution recovery $(128 \times 128$ matrix, $4.42 \times 4.42 \mathrm{~mm}$ pixel) without noise reduction filter. The various combinations of the number of subsets and iterations were 10 (fixed) and 1-10, respectively. The quantitative analysis software accompanying the SPECT/CT system used in this study was designed to calculate quantitative values without noise reduction filters. Therefore, all SPECT image quality evaluations were performed using SPECT images without noise reduction filters.

\section{System planar sensitivity}

The system planar sensitivity $[17,21]$ was measured using the automatic mode provided by the manufacturer (GE Healthcare). A plastic Petri dish with ${ }^{99 \mathrm{~m}} \mathrm{Tc}$ solution $(124.4 \mathrm{MBq}$ ) was placed on styrene foam (10-cm thickness) at the collimator surface, and the image was acquired from the anterior and posterior views. The system planar sensitivity was calculated using the following formula:

system planar sensitivity $(\mathrm{cps} / \mathrm{MBq})=\mathrm{T}_{\text {count }} / \mathrm{AT},(1)$

where $\mathrm{A}$ is the decay corrected activity at the start time of acquisition, $\mathrm{T}$ is the acquisition time, and $\mathrm{T}_{\text {count }}$ is the average of the total counts in the anterior and posterior images. The measured system planar sensitivity was $88.4 \mathrm{cps} / \mathrm{MBq}$.

\section{Evaluation of image quality and quantification}

SPECT images from each acquisition method were compared in terms of normal bone coefficient of variance (CV), contrast-to-noise ratio (CNR), full width at half maximum (FWHM), and recovery coefficient (RC). Optimized number of iterations was determined from CV and CNR. For measuring image quality indices, a circular region of interest (ROI) with $80 \%$ of the diameter was set in five slices (Fig. 2), including the normal bone portion of the axial image. Similarly, ROls were set in a slice with the largest diameter of the $17 \mathrm{~mm}$ diameter tumor area. ROI settings were based on CT images. The mean count and standard deviation were obtained from the tumor and normal area ROIs. The CV was calculated using the following formula:

$$
C V=\frac{C_{S D}}{C_{\text {normal }}}
$$


where $\mathrm{C}_{\text {normal }}$ and $\mathrm{C}_{\mathrm{SD}}$ represent the mean count and standard deviation of the normal bone area, respectively.

The CNR was calculated using the following formula:

$$
C N R=\frac{C_{\text {tumor }}-C_{\text {normal }}}{C_{S D}}
$$

3

where $\mathrm{C}_{\text {tumor }}$ is the mean count of the tumor area.

The FWHM was calculated from the profile curve of the spinous process (Fig. 2) using Prominence processor version 3.1 software (http://nm.jsrt.or.jp/blog.html).

For calculating RC, a sphere shape voxel of interest was set at $80 \%$ size at each tumor bone area using software (Q. Metrix, GE Healthcare), and the following formula was used to calculate RC.

$$
R C(\%)=\frac{A_{\text {SPECT }}}{A_{\text {True }}} \times 100
$$

4

where $A_{\text {True }}$ is the radioactivity concentration enclosed in the tumor bone area of the phantom, and $A_{S P E C T}$ is the radioactivity concentration in the tumor bone area obtained from the SPECT image. Here, $A_{\text {True }}$ is $300 \mathrm{kBq} / \mathrm{mL}$.

\section{Results}

\section{CV}

Figure 3 shows the CV of SwiftScan and SSM at different iterations. The CV increased with increasing number of iterations, but it decreased with increasing acquisition time. In the short-time acquisition (7 min or less), the CV tended to increase monotonically, but in the long-time acquisition (17 min or more), the lowest CV was observed when the number of iterations was 5. Comparing SwiftScan and SSM when the subset was 10 and the number of iterations was fixed at 5, CV of SSM was higher in the short-time acquisition but similar in a long-time acquisition (Table 1). 
Table 1

Comparison of CV between SwiftScan and SSM.

\begin{tabular}{|lll|}
\hline Acquisition time (min) & \multicolumn{2}{l|}{ CV } \\
\cline { 2 - 3 } & SwiftScan & SSM \\
\hline 5 & 0.15 & 0.18 \\
\hline 7 & 0.14 & 0.16 \\
\hline 17 & 0.12 & 0.11 \\
\hline 32 & 0.10 & 0.10 \\
\hline CV coefficient of variance, SSM step and shoot \\
\hline
\end{tabular}

CNR

Figure 4 shows the change in CNR of SwiftScan and SSM at different iterations. The CNR of both acquisition methods increased with increasing iteration, reaching its highest value at the fifth iteration, and tended to decrease after that. Comparison of the CNR of SwiftScan and SSM with subset 10 and iteration fixed at 5 is shown in Table 2. The CNR of SwiftScan was higher than that of SSM in short-time acquisition. However, the CNR of SSM was equivocal or higher than that of SwiftScan in long-time acquisition.

From the CV and CNR results, optimal reconstruction parameters were fixed at numbers of subsets 10 and iterations 5 . Figure 5 shows the phantom images for each acquisition time. In both acquisition methods, noise is noticeable at short-time acquisition, but the image quality improves as the acquisition time increases. There was no visually definite difference between the phantom images by SwiftScan and SSM over 7 min acquisition time. However, the shape of the tumor area using SwiftScan was different than that using SSM at 5 min acquisition.

Table 2

Comparison of CNR between SwiftScan and SSM.

\begin{tabular}{|lll|}
\hline Acquisition time (min) & \multicolumn{2}{l|}{ CNR } \\
\cline { 2 - 3 } & SwiftScan & SSM \\
\hline 5 & 5.01 & 3.55 \\
\hline 7 & 6.13 & 5.65 \\
\hline 17 & 13.8 & 15.7 \\
\hline 32 & 15.8 & 15.4 \\
\hline \multicolumn{4}{|l}{ CNR contrast-to-noise ratio, SSM step and shoot } \\
\hline
\end{tabular}

FWHM 
A comparison of FWHM for SwiftScan and SSM is shown in Table 3. The FWHM for SwiftScan (15.4$15.9 \mathrm{~mm})$ and SSM (15.2-16.5 mm) were almost constant when the acquisition time was $7 \mathrm{~min}$ or longer. However, the FWHM for SwiftScan $(22.6 \mathrm{~mm})$ and SSM $(18.6 \mathrm{~mm})$ were higher at $5 \mathrm{~min}$ acquisition, deviating from the actual size $(10 \mathrm{~mm})$.

Table 3

Comparison of FWHM between SwiftScan and SSM.

\begin{tabular}{|lll|}
\hline Acquisition time (min) & \multicolumn{2}{l|}{ FWHM (mm) } \\
\cline { 2 - 3 } & SwiftScan & SSM \\
\hline 5 & 22.6 & 18.6 \\
\hline 7 & 15.4 & 15.2 \\
\hline 17 & 15.9 & 16.5 \\
\hline 32 & 15.8 & 15.4 \\
\hline FWHM full width at half maximum, SSM step and shoot \\
\hline
\end{tabular}

RC

Figure 6 shows the relationship between RC for each diameter of tumor bone area and acquisition times. RC of both SwiftScan and SSM decreased as the diameter of the tumor area decreased. Compared to the RC of SwiftScan and SSM at the same acquisition time, a difference was observed when the small tumor diameter (13 and $17 \mathrm{~mm}$ ) at the short-time acquisition (5 and $7 \mathrm{~min}$ ). Furthermore, in the small tumor diameter, the short-time acquisition showed a tendency of higher RC than that of long-time acquisition. RCs of $17 \mathrm{~mm}$ diameter for SwiftScan and SSM were as follows: $5 \mathrm{~min}: 84.8 \%, 80.7 \% ; 7 \mathrm{~min}: 86.1 \%$, $90.6 \%$; $17 \mathrm{~min}: 72.4 \%, 72.1 \%, 32 \mathrm{~min}: 73.8 \%, 72.6 \%$.

\section{Discussion}

In this study, the properties of SwiftScan in quantitative bone SPECT were investigated using a phantom. The CV and CNR of SwiftScan tended to be superior to those of SSM in short acquisition time. However, in long acquisition times, CV of SwiftScan and SSM were comparable, whereas the CNR of SSM was higher than that of SwiftScan. SwiftScan and SSM showed almost the same RC, regardless of the acquisition time in terms of quantitative performance. These results suggest that SwiftScan provides the same or better image quality and quantitativeness as SSM in short acquisition time quantitative bone SPECT examination.

The CV of SwiftScan was lower than that of SSM for short-time acquisition, but they were almost equal for long-time acquisition. The reason for this can be explained by the fact that SwiftScan adds the count 
of detector moving time (approximately $4 \mathrm{~s} /$ view, total $120 \mathrm{~s}$ ) to the SSM. When the SSM is at $5 \mathrm{~min}$ acquisition ( $6 \mathrm{~s} / \mathrm{view}$ for SSM), SwiftScan increases the count by 1.67 times ( $6 \mathrm{~s} / \mathrm{view}+4 \mathrm{~s} / \mathrm{view})$, whereas when the SSM is at $32 \mathrm{~min}$ acquisition ( $60 \mathrm{~s} / \mathrm{view})$, SwiftScan only increases the count by about 1.07 times $(60 \mathrm{~s} / \mathrm{view}+4 \mathrm{~s} / \mathrm{view})$. Cao et al. [22] compared the image quality of simulated continuous SSM (CSSM) with that of SSM through simulation experiments, and they showed that CSSM is almost the same as SSM for long-time acquisition, but CSSM substantially improved for short-time acquisition.

The CNR of SwiftScan was higher than that of SSM in short-time acquisition due to improved contrast in the tumor area. However, the CNR of SSM was equivocal or higher than that of SwiftScan in long-time acquisition. The reason for this is the same as that for CV: in the short-time acquisition, the effect of the added counts during detector movement in SwiftScan is significant. The acquisition parameters, such as the number of projections and acquisition time, are less relevant in regions with high-count statistics (e.g., in the tumor area) compared with regions with low-count statistics (e.g., in the background) [13]. Therefore, SwiftScan is more advantageous than SSM in short-time acquisition because the image quality can be improved by adding the counts of detector moving time. However, for long acquisition time, there might be no advantage in using SwiftScan instead of SSM.

In the FWHM evaluation, SSM and SwiftScan deviated from the actual size $(10 \mathrm{~mm})$ at $5 \mathrm{~min}$ acquisition $(6 \mathrm{~s} / \mathrm{view})$. This deviation may be owing to insufficient convergence of the reconstruction owing to high noise in the short acquisition time. Furthermore, SwiftScan may have increased the FWHM due to the addition of counts during detector movement. Such an increase in FWHM (degradation of resolution) may affect the region of interest settings and the reliability of quantitative value.

In quantitative value comparison, SwiftScan and SSM were similar. However, in both cases, the RC was higher for smaller tumor diameters in short-time acquisition. The quantitative analysis software accompanying the SPECT/CT system used in this study was designed to calculate quantitative values without noise reduction filters. Therefore, the noise reduction filter was not used for all SPECT images. Thus, the quantitative values may have been estimated high due to the extremely high noise in the shorttime acquisition and the significant effect of noise in the ROI due to the small tumor diameter. The shorttime acquisition images without noise reduction are insufficient for reading by diagnosticians due to the high noise. Kupitz et al. [13] recommended using two different reconstruction protocols with task-specific optimized setups (quantitative and non-quantitative). Based on the results of CV and CNR, the number of iterations was set to 5 . It is said that increasing the number of iterations converges the quantitative values to a known value [23]. In this study, the quantitative value of the reference and other tumor parts did not change with the number of iterations (data are not shown). However, note that an increase in the number of iterations increases noise.

In quantitative bone SPECT, attempts to reduce acquisition time are being investigated. Ichikawa et al. [24] showed that the XSPECT bone reconstruction algorithm could maintain high image quality and quantitativeness even with a 3-min acquisition time. Although the reconstruction algorithm currently depends on the vendor, there is a possibility of further shortening of acquisition time and/or improving 
image quality and quantitativeness by applying a reconstruction algorithm, such as XSPECT bone to SwiftScan. Moreover, SwiftScan may reduce the total examination time if the image quality and quantitativeness are sufficient. Shortening the total examination time and obtaining quantitative and image quality comparable to that of SSM would reduce the possibility of patient motion and mental and physical distress.

This study has several limitations. First, the effect of the change in the acquisition time and radioactive concentration level has not been investigated in detail because it is a phantom experiment. Therefore, it is necessary to investigate the detailed acquisition time variation by simulation. Second, the lack of comparison with the CM acquisition. Since continuous acquisition is used in many SPECT/CT devices, a comparison is necessary. Lastly, there is a lack of clinical study. It is necessary to examine whether the results of phantom experiments are equivalent to those in clinical practice.

\section{Conclusions}

SwiftScan in quantitative bone SPECT provides improved CV and CNR in short acquisition times (less than $7 \mathrm{~min}$ ) with quantitativeness similar to SSM. Therefore, using SwiftScan in clinical settings instead of the SSM scan protocol in short-time acquisition (less than $7 \mathrm{~min}$ ) might provide higher quality diagnostic images. Our results would provide important information for the use of SwiftScan on quantitative bone SPECT.

\section{Abbreviations}

SPECT: Single-photon emission computed tomography; SSM: Step and shoot mode; CV: Coefficients of variance; CNR: Contrast-to-noise ratio; $\mathrm{CT}$ : computed tomography; FWHM: Full width at half maximum; RC: Recovery coefficients; SUV: Standardized uptake value; AC: Attenuation correction; SC: Scatter correction; SPECT/CT: Single-photon emission computed tomography/computed tomography; OSEM: Ordered subset expectation maximization; ROI: Region of interest

\section{Declarations}

\section{Ethics approval and consent to participate}

Not applicable

\section{Consent for publication}

Not applicable

\section{Availability of data and material}

SPECT and CT raw datasets are available from the corresponding author on reasonable request. All data analyzed during this study are included in this published article. 


\section{Competing interests}

The authors declare that they have no competing interests.

\section{Funding}

Not applicable

\section{Authors' contributions}

The conceptualization of this study was conducted by TS and YS. Methodology was developed by TS, and the measurements were performed by ST, NS, and YI. The analysis of the data was done by TS and ST. The original draft was written by TS, and revision was done by YK, YK, TT, and TI. The draft was reviewed and edited by all authors. The authors read and approved the final manuscript.

\section{Acknowledgments}

The authors are grateful to Kenji Yoshida and Hideyasu Hosono (GE Healthcare, Japan) for their useful suggestions and supports.

\section{References}

1. Langsteger W, Rezaee A, Pirich C, Beheshti M. ${ }^{18} \mathrm{~F}-\mathrm{NaF}-\mathrm{PET} / \mathrm{CT}$ and ${ }^{99 \mathrm{~m}} \mathrm{Tc}-\mathrm{MDP}$ bone scintigraphy in the detection of bone metastases in prostate cancer. Semin Nucl Med. 2016;46:491-501.

2. Hamaoka T, Madewell JE, Podoloff DA, Hortobagyi GN, Ueno NT. Bone imaging in metastatic breast cancer. J Clin Oncol. 2004;22:2942-53.

3. Van den Wyngaert T, Strobel K, Kampen WU, Kuwert T, van der Bruggen W, Mohan HK, et al. The EANM practice guidelines for bone scintigraphy. Eur J Nucl Med Mol Imaging. 2016;43:1723-38.

4. Utsunomiya D, Shiraishi S, Imuta M, Tomiguchi S, Kawanaka K, Morishita S, et al. Added value of SPECT/CT fusion in assessing suspected bone metastasis: comparison with scintigraphy alone and nonfused scintigraphy and CT. Radiology. 2006;238:264-71.

5. Israel O, Pellet O, Biassoni L, De Palma D, Estrada-Lobato E, Gnanasegaran G, et al. Two decades of SPECT/CT - the coming of age of a technology: An updated review of literature evidence. Eur $\mathrm{J}$ Nucl Med Mol Imaging. 2019;46:1990-2012.

6. Savelli G, Maffioli L, Maccauro M, De Deckere E, Bombardieri E. Bone scintigraphy and the added value of SPECT (single photon emission tomography) in detecting skeletal lesions. Q J Nucl Med. 2001;45:27-37.

7. Palmedo H, Marx C, Ebert A, Kreft B, Ko Y, Türler A, et al. Whole-body SPECT/CT for bone scintigraphy: diagnostic value and effect on patient management in oncological patients. Eur $\mathrm{J}$ Nucl Med Mol Imaging. 2014;41:59-67. Available from: http://link.springer.com/10.1007/s00259-0132532-6. 
8. Cachovan M, Vija A, Hornegger J, Kuwert T. Quantification of ${ }^{99 \mathrm{~m}} \mathrm{Tc}$-DPD concentration in the lumbar spine with SPECT/CT. EJNMMI Res. 2013;3:45.

9. Bailey DL, Willowson KP. An Evidence-Based Review of Quantitative SPECT Imaging and potential clinical applications. J Nucl Med. 2013;54:83-9.

10. Miyaji N, Miwa K, Tokiwa A, Ichikawa H, Terauchi T, Koizumi M, et al. Phantom and clinical evaluation of bone SPECT/CT image reconstruction with XSPECT algorithm. EJNMMI Res. 2020;10:71.

11. Hishikawa M, Matsutomo N, Yamamoto T. Impact of reconstruction parameters on quantitative bone SPECT imaging: A novel thoracic spine phantom study. Hellenic J Nucl Med. 2019;22 Suppl 2:140. Available from: http://www.ncbi.nlm.nih.gov/pubmed/31802053.

12. Ichikawa $H$, Onoguchi $M$, Shibutani $T$, Kato $T$, Ito $T$, Shimada $H$. Optimization of cross-calibration factor for quantitative bone SPECT without attenuation and scatter correction in the lumbar spine: head-to-head comparison with attenuation and scatter correction. Nucl Med Commun. 2021;42:1404-16.

13. Kupitz D, Wissel H, Wuestemann J, Bluemel S, Pech M, Amthauer H, et al. Optimization of SPECT/CT imaging protocols for quantitative and qualitative ${ }^{99 m}$ Tc SPECT. EJNMMI Phys. 2021;8:57. Available from: https://ejnmmiphys.springeropen.com/articles/10.1186/s40658-021-00405-3.

14. Bieszk JA, Hawman EG. Evaluation of SPECT angular sampling effects: continuous versus step-andshoot acquisition. J Nucl Med. 1987;28:1308-14.

15. Shibutani T, Onoguchi M, Naoi Y, Yoneyama H, Konishi T, Tatami R, et al. The usefulness of SwiftScan technology for bone scintigraphy using a novel anthropomorphic phantom. Sci Rep. 2021;11:2644.

16. Bailly M, le Rouzic G, Metrard G, Ribeiro MJ. Faster Acquisition for Dopamine Transporter Imaging Using Swiftscan Step and Shoot Continuous SPECT Without Impairing Visual and Semiquantitative Analysis. Front Med (Lausanne). 2020;7. Available from: https://www.frontiersin.org/article/10.3389/fmed.2020.00235/full.

17. Thibault F, Bailly M, Le Rouzic G, Metrard G. Clinical evaluation of General Electric new Swiftscan solution in bone scintigraphy on Nal-camera: A head to head comparison with Siemens Symbia. PLOS ONE. 2019;14:e0222490.

18. Shibutani T, Onoguchi M, Yoneyama H, Konishi T, Nakajima K. Performance of SwiftScan planar and SPECT technology using low-energy high-resolution and sensitivity collimator compared with Siemens SPECT system. Nucl Med Commun. 2021;42:732-7.

19. Picone V, Makris N, Boutevin F, Roy S, Playe M, Soussan M. Clinical validation of time reduction strategy in continuous step-and-shoot mode during SPECT acquisition. EJNMMI Phys. 2021;8:10.

20. Ichikawa H, Kawakami K, Onoguchi M, Shibutani T, Nagatake K, Hosoya T, et al. Automatic quantification package (Hone Graph) for phantom-based image quality assessment in bone SPECT: computerized automatic classification of detectability. Ann Nucl Med. 2021;35:937-46. 
21. Matsutomo N, Matsumoto S, Yamamoto T, Sato E. Validation of a calibration method using the cross-calibration factor and system planar sensitivity in quantitative single-photon emission computed tomography imaging. Radiol Phys Technol. 2017;10:439-45.

22. Cao Z, Maunoury C, Chen CC, Holder LE. Comparison of continuous step-and-shoot versus step-andshoot acquisition SPECT. J Nucl Med. 1996;37:2037-40. Available from:

http://www.ncbi.nlm.nih.gov/pubmed/8970530.

23. Jaskowiak CJ, Bianco JA, Perlman SB, Fine JP. Influence of reconstruction iterations on ${ }^{18} \mathrm{~F}$-FDG PET/CT standardized uptake values. J Nucl Med. 2005;46:424-8.

24. Ichikawa H, Miyaji N, Onoguchi M, Shibutani T, Nagaki A, Kato T, et al. Feasibility of ultra-high-speed acquisition in XSPECT bone algorithm: a phantom study with advanced bone SPECT-specific phantom. Ann Nucl Med. 2021. doi:10.1007/s12149-021-01689-2.

\section{Figures}

\section{Figure 1}

Overview of the $\mathrm{SIM}^{2}$ phantom and enclosed radioactivity concentration.

\section{Figure 2}

Settings of the region of interest for evaluating image qualities.

\section{Figure 3}

Relationship between update number and coefficient of variation (CV). CV of various acquisition times using SwiftScan (a) and step and shoot (SSM) (b). The update number is the product of the subset and iterations.

\section{Figure 4}

Relationship between update number and contrast-to-noise ratio (CNR). CNR of various acquisition times using SwiftScan (a) and step and shoot (SSM) (b). The update number is the product of the subset and the iterations. 


\section{Figure 5}

The phantom images of various acquisition time using SwiftScan and step and shoot (SSM). The upper row shows SwiftScan, and the lower row shows SSM.

\section{Figure 6}

Recovery coefficients for each diameter of tumor bone area at various acquisition times. Circles indicate SwiftScan, and cross marks indicate step and shoot (SSM). 